\title{
Poster PD-057
}

Treatment of a vulvar Paget disease

by photodynamic therapy with a

new light emitting fabric based

device

C. Vicentini ${ }^{1,2, *}$, O. Carpentier ${ }^{1}$, J.-B. Tylcz ${ }^{2}$, L. Mortier ${ }^{1,2,3}, \mathrm{~N}$.

Betrouni $^{2,3}$, S. Mordon ${ }^{2,3}$

${ }^{1}$ Clinic of Dermatology, Lille University

Hospital, France

${ }^{2}$ ONCO-THAI INSERM U1189, Lille, France

${ }^{3}$ Lille University, France

Introduction: The extra-mammary Paget disease is an intraepi-dermal carcinoma with glandular characteristics. The standard treatment is surgical excision in depth. According to the anatomi-cal sites, this technic is not always appropriate and photodynamic therapy (PDT) can be an alternative choice. However, the major side effect of PDT is pain, which is sometimes leading to the discontin-uation of treatment. The light emitting fabric is a part of a device that is under clinical evaluation for the treatment of actinic kerato-sis with PDT. We report the observation of a vulvar Paget disease treated by this mean with an excellent tolerance.

Clinical observation: The patient has been diagnosed with vul-var Paget disease for 25 years. The disease recurred constantly despite several imiquimod applications and LASER treatments. The patient refused the renewal of these methods due to their poor tolerance. The PDT treatment with a conventional device was promptly interrupted because of a strong pain. We performed 3 PDT sessions a month apart with the light emitting fabric at low irradiance with a good result and an excellent tolerance.

Discussion: There are no controlled trials evaluating the effi-cacy of PDT in the treatment of extra-mammary Paget disease. The treatment and follow-up protocols in the literature are heteroge-neous. Pain is the most common side effect with greater intensity for perineal locations. PDT for this location is impractical outside of anesthesia or hypnosis. A specific study is required but the light emitting fabric could be indicated for the treatment of Paget disease of perineal location.

Conclusion: We report the case of a vulvarPaget disease treated with efficacy and an excellent tolerance by the new light emitting fabric device. 